\title{
Radioembolization in Metastatic Breast Cancer
}

\author{
Amy Deipolyi, MD, $\mathrm{PhD}^{1}$ Yolanda Bryce, $\mathrm{MD}^{1}$ (1) Ripal Gandhi, MD² \\ ${ }^{1}$ Memorial Sloan Kettering Cancer Center, New York, New York \\ 2 Miami Cardiac and Vascular Institute, Miami Cancer Institute, Miami, \\ Florida \\ Address for correspondence Yolanda Bryce, MD, Memorial Sloan \\ Kettering Cancer Center, 1275 York Avenue, New York, NY 10065 \\ (e-mail: brycey@mskcc.org; ycdbryce@gmail.com).
}

Dig Dis Interv 2021;5:113-117.

\begin{abstract}
Keywords

- metastatic breast cancer

- radioembolization

- metastatic disease

Metastatic breast cancer (MBC) remains the second cause of cancer death in women, despite improvements in early breast cancer detection and treatments, with a 5-year survival of only $27 \%$. Patients with MBC involving the liver have a 5 -year survival of only 3.8 to $12 \%$. Systemic therapy is the cornerstone for the treatment of $M B C$ according to the National Comprehensive Cancer Network (NCCN) guidelines. Radioembolization is not specifically prescribed by the NCCN guidelines in the treatment of MBC liver metastasis, but is an emerging therapy with some promising results. The two primary reasons to offer radioembolization would be to prolong life and to palliate and improve quality of life. We review here the indications, contraindications, technique, case examples, and unanswered questions.
\end{abstract}

\section{Scope of Metastatic Breast Cancer}

Metastatic breast cancer (MBC) remains the second cause of cancer death in women, despite improvements in early breast cancer detection and treatments, with a 5-year survival of only $27 \%{ }^{1}$ A subset of $\mathrm{MBC}$, triple negative $\mathrm{MBC}$ (TNMBC) has particularly poor prognosis with a 5 -year survival of $11 \%$ and a median survival of only 1 year. ${ }^{2}$ About 6 to $10 \%$ of breast cancer patients present with de novo metastases (metastatic disease at the time of initial presentation) and nearly $30 \%$ of early breast cancer will progress to metastatic disease. ${ }^{3,4}$

The most common sites of metastases in breast cancer are liver, lung, bone, and brain, with liver comprising $50 \%$ of metastatic cases. ${ }^{5}$ In addition, 5 to $12 \%$ of patients have liver metastases as the primary site of breast cancer recurrence. ${ }^{5}$ Left untreated, survival is poor in patients with liver metastases, ranging from 4 to 8 months. ${ }^{6}$ In patients with de novo metastatic disease to the liver, median overall survival is only 15 months, and 8 months in the setting of triple negative breast cancer. ${ }^{7}$ Overall, patients with $\mathrm{MBC}$ involving the liver have a 5 -year survival of only 3.8 to $12 \%{ }^{7}$

received

November 19, 2020 accepted after revision February 16, 2021
Issue Theme Y-90; Guest Editors, Ripal T. Gandhi, MD, FSIR, FSVM and Suvranu Ganguli, MD, FSIR

\section{Standard of Care for Metastatic Breast Cancer}

Breast cancer is composed of several molecular subtypes that show marked differences in tumor biology and clinical behavior. Moreover, hormone expression and HER2neu status may be discordant between primary tumors and metastatic disease and loss of therapeutic target may also develop between metastatic sites. ${ }^{8}$ Generally, systemic therapy is based on endocrine therapy for hormone-positive disease, chemotherapy for TNMBC-with new addition of immunotherapy with atezolizumab, and anti-HER2neu gene antibodies such as trastuzumab or pertuzumab for HER2neu-positive disease. PARP inhibitors, PI3K inhibitors, and CDK4/6 inhibitors are novel with promising results. ${ }^{8}$ However, given the particular poor prognosis of liver metastases despite optimal systemic therapy and given resection of liver metastases in breast cancer is typically not feasible due to the presence of multisegmental liver disease at the time of diagnosis, locoregional therapy may be justified. $^{9}$ (c) 2021. Thieme. All rights reserved. Thieme Medical Publishers, Inc., 333 Seventh Avenue, 18th Floor, New York, NY 10001, USA
DOI https://doi.org/ 10.1055/s-0041-1727185. ISSN 2472-8721. 


\section{Indications and Contraindications}

Radioembolization is not specifically prescribed by the National Comprehensive Cancer Network (NCCN) guidelines in the treatment of MBC liver metastasis, but is an emerging therapy with some promising results. The two primary reasons to offer radioembolization would be to prolong life and to palliate and improve quality of life. Locoregional therapy with external radiation to oligometastatic disease has been shown to increase survival in a randomized trial, though similar studies have not been conducted for embolotherapies. Several studies have shown that median survival after radioembolization of breast cancer liver metastasis is approximately 1 year, while these are retrospective studies, these data are promising because typically the patients have refractory cancer and have exhausted systemic therapy options. Patients who have progressive liver metastases in the setting of stable or decreasing extrahepatic metastases are candidates for hepatic radioembolization, with the intent to prolong the utility of the patient's current systemic therapy regimen, preserving other lines of therapy for future use. This theoretical benefit has not been explicitly studied in the context of breast cancer. Finally, liver metastases can be painful; radioembolization may reduce growth and size of hepatic tumors, palliating symptoms.

Absolute contraindications to radioembolization include contraindication to angiography and visceral catheterization (e.g., uncorrectable coagulopathy or anaphylactic reaction to contrast), life-threatening comorbidity, progressive extrahepatic metastasis thought to immediately threaten survival, pulmonary insufficiency or clinically evident chronic obstructive pulmonary disease, and liver failure. In regard to liver failure, careful evaluation of preexisting hepatic function is important in patient selection. Evidence of portal hypertension, including more than trace ascites or history of hepatic encephalopathy, precludes patients from undergoing radioembolization which can worsen liver function. A relatively strict total bilirubin cutoff of $2.0 \mathrm{mg} / \mathrm{dL}$ is typically used, though any rapid increase in bilirubin prior to radioembolization should delay treatment until hepatic function improves or stabilizes. When large volume/lobar treatments are planned, patients with tumor burden of 10 to $70 \%$ are considered reasonable candidates; historically, tumor replacement exceeding $70 \%$ of liver parenchyma has been considered a contraindication, primarily due to microsphere manufacturer guidelines. ECOG status of 2 or greater is a contraindication to radioembolization. Finally, prior bilioenteric anastomosis or other procedure that disrupts the ampulla leading to bacterial colonization of the bile ducts is a relative contraindication, as radioembolization can lead to infectious biliary complications at a rate of $8 \%$ in this population. The latter group of patients may be safely treated with antibiotic prophylaxis (with or without bowel preparation) before and after radioembolization. ${ }^{10}$

\section{Technique}

Radioembolization of breast cancer liver metastasis most commonly involves large volume/lobar infusions of radioac- tive microspheres, and infrequently may involve segmental infusions for more localized disease. Patients undergo a preliminary mapping procedure to calculate the lung shunt fraction, which is typically low $(<10 \%)$, and to define target vessels. Most commonly, patients will then undergo two lobar infusions separated by at least 4 to 6 weeks to allow for recovery of liver function (-Fig. $\mathbf{1}$ ). Like colorectal cancer, breast cancer is less arterially enhancing than hepatocellular carcinoma, which may in turn entail higher radiation doses to normal liver parenchyma. In these cases, dose reduction and prolonging the interval between lobar treatments are strategies that might mitigate deleterious effects on hepatic function.

When localized microsphere infusions are planned, as in cases of radiation segmentectomy, the target volume can be ascertained from the mapping procedure and a dose calculated to exceed approximately $200 \mathrm{~Gy}$, based on prior literature primarily in the context of hepatocellular carcinoma. Newer data suggest that the new threshold ablative dose for hepatocellular carcinoma radiation segmentectomy is more than 400 gray, which was showed to result in complete pathologic necrosis. ${ }^{11}$ It may be necessary to use large particle bland embolization, such as with Gel Foam slurry, to "protect" areas distal to target vessels and boost the

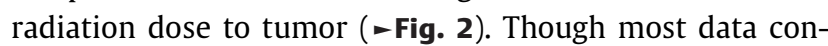
cerning radiation segmentectomy are regarding hepatocellular carcinoma, one small study that included one patient with breast cancer suggested high objective response and low complication rates.

\section{Reported Outcomes}

Outcomes reported after radioembolization include survival statistics, imaging response, and toxicities. Like for other embolotherapies, imaging response after radioembolization is well correlated with overall survival and may therefore be considered a surrogate marker of survival. Medial overall survival after treatment ranges from 6 to 13 months, likely reflecting differences in patient selection by institution. Reported imaging response is also variable, and reflects differences in response to evaluation criteria. By RECIST criteria, disease control rates range from approximately 70 to more than $90 \%$. As in colorectal cancer, metabolic imaging appears more sensitive to response, with objective response rates of $70 \%$ or higher. Imaging response by PERCIST criteria is seen by 1 to 2 months and persists until at least 6 months.

Toxicities include complications such as nontarget embolization and negative impact on liver function, the worst of which manifests as radioembolization-induced liver failure. Radioembolization of breast cancer liver metastasis has been reported to carry less than $15 \%$ of grade 3 and higher adverse events. Higher pre-radioembolization baseline bilirubin levels are associated with grade 3 and greater hepatotoxicity after treatment. These findings highlight the importance of assessing baseline hepatic function when evaluating patients' candidacy for radioembolization. 

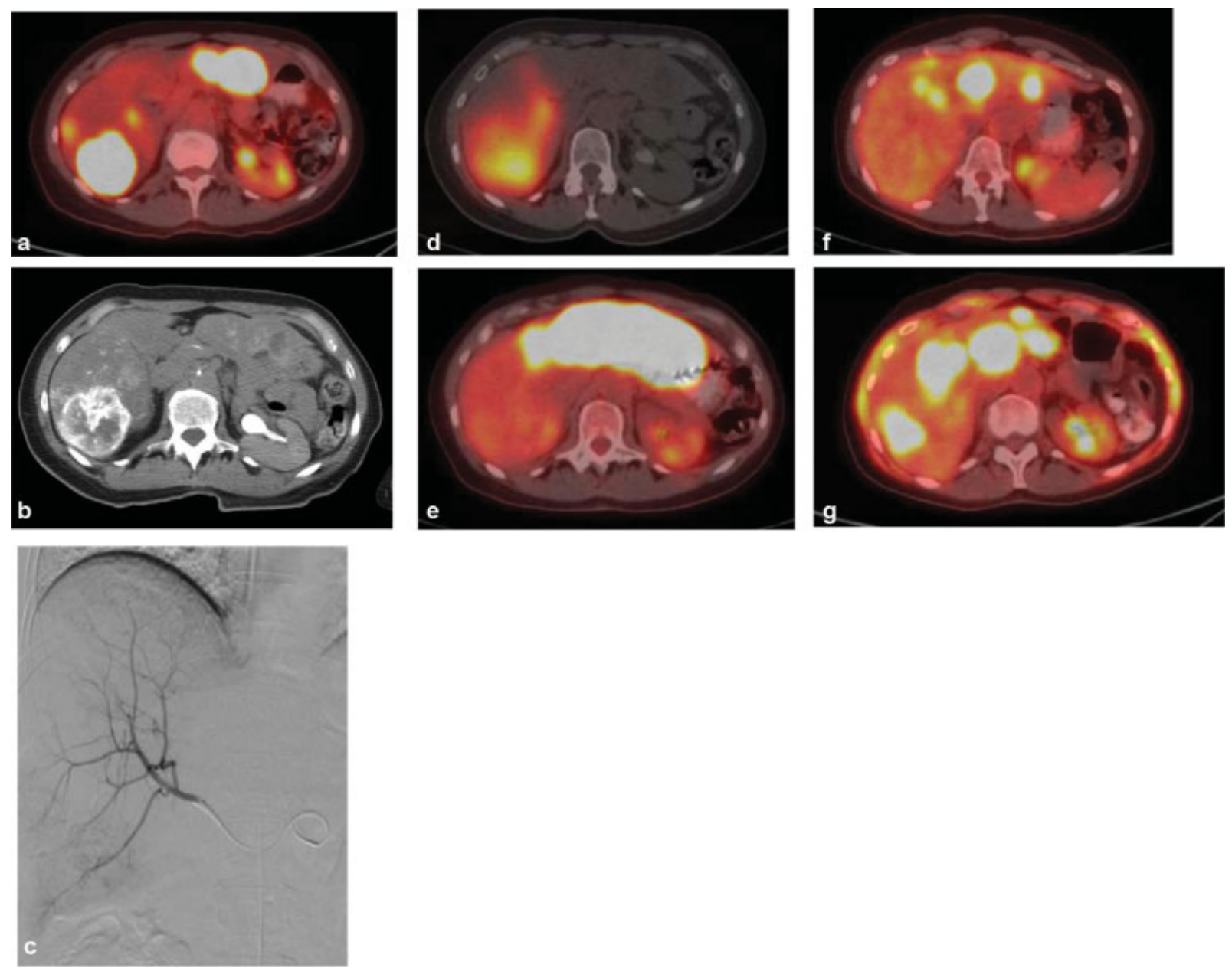

Fig. 1 Radioembolization of multifocal bilobar breast cancer liver metastasis. (a) PET/CT in a 57-year-old woman with metastatic breast cancer demonstrates bilobar hepatic metastases that were progressing, while extrahepatic metastases were stable or decreasing. (b) Given the multifocality of lesions, two lobar treatments were planned; intraprocedural CTA demonstrates the territory of the right hepatic treatment. (c) Right lobar radioembolization was performed first, as the bulk of disease was present in the right lobe. Glass microspheres were used, with a target dose of $120 \mathrm{~Gy}$. (d) Preferential uptake into tumor was noted on the post-administration bremsstrahlung SPECT/CT. (e) PET/CT 2 months later demonstrates a complete response in the treated right lobe, with progression of disease in the left lobe. (f) The patient subsequently underwent left lobar radioembolization, with partial response on PET/CT. (g) By 9 months after the initial right lobe radioembolization, the patient had developed new lesions in both hepatic lobes and in extrahepatic sites. However, locoregional therapy to progressive liver metastasis in the patient delayed a change in systemic therapy by several months.

The other primary embolotherapy offered for metastatic breast cancer is transarterial chemoembolization (TACE). One retrospective single-institution study comparing TACE and radioembolization for $\mathrm{MBC}$ reported better imaging outcomes and lower complication rates for radioembolization compared with TACE. Comparing the two modalities, several retrospective studies show better imaging outcomes for radioembolization; survival outcomes have wide variability between centers. Given that radioembolization yields better imaging outcomes, which are associated with longer survival and lower complication rates, and that radioembolization is associated with better quality of life and lower hospitalization rates compared with TACE in the context of hepatocellular carcinoma, it is reasonable to offer radioembolization preferentially to TACE as a locoregional therapy for breast cancer liver metastasis.

\section{Unanswered Questions}

There are many unanswered questions regarding utilization of radioembolization in patients with MBC. While many of the existing studies of radioembolization have been done in the setting of chemorefractory disease/salvage therapy, the optimal timing of liver-directed therapy is not known. One study suggests that radioembolization within 6 months of diagnosis of hepatic metastatic disease results in improved overall survival; however, more clinical data are needed to help guide the timing of radioembolization with respect to the line of systemic therapy. ${ }^{12}$ It is not definitively known if outcomes are better if radioembolization is offered earlier during the course of a patient's disease. Whether systemic therapy should be held and for how long prior to radioembolization is unclear. The patient's receptor status may 

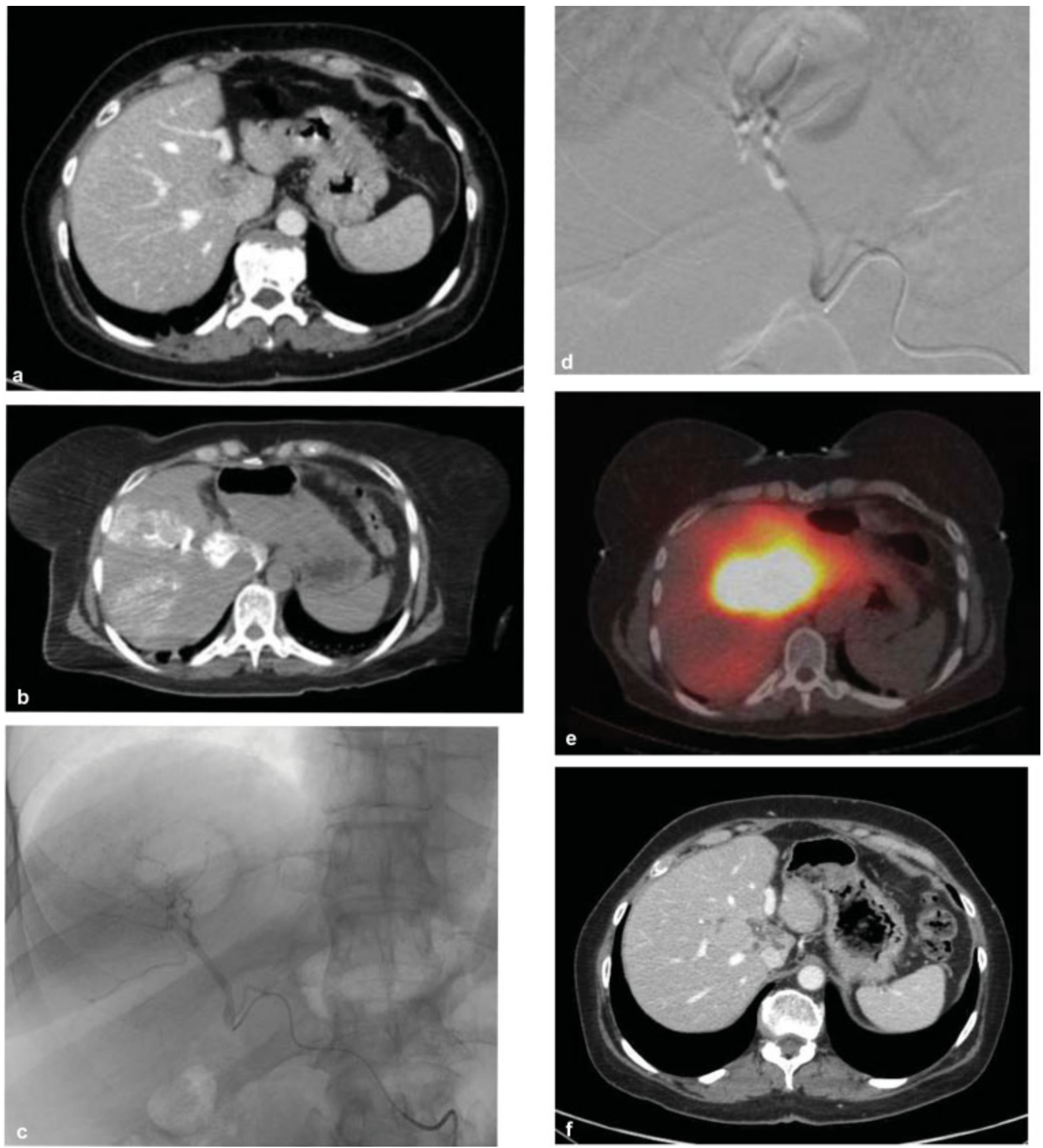

Fig. 2 Radiation segmentectomy for caudate breast cancer metastasis. (a) Contrast-enhanced CT in a 45-year-old woman with metastatic breast cancer demonstrates an enlarging caudate tumor, without any right lobe lesions, in the absence of extrahepatic metastatic disease. The lesion was not considered amenable to thermal ablation given its size and location. (b) Intraprocedural CTA during the mapping demonstrates that the caudate is supplied by the anterior right hepatic artery. (c) The caudate artery is the first, medially traversing, branch of the anterior right hepatic artery. The vessel could not be directly cannulated despite attempting directional microcatheter deployment. (d) On the day of treatment, the right anterior hepatic artery was embolized with Gel Foam slurry distal to the caudate artery takeoff, to redirect flow of microspheres to the caudate vessel. (e) A dose calculated for the right lobe was delivered after embolization, with dense uptake in the caudate noted on the postadministration bremsstrahlung SPECT/CT. (f) Follow-up contrast-enhanced CT 8 weeks later shows complete response in the caudate tumor.

be helpful to guide utilization of liver-directed therapy. Estrogen receptor positivity appears to be a positive predictor of prolonged survival, but there is paucity of solid evidence. There may be a synergistic effect of systemic therapy and radioembolization, but this hypothesis requires further exploration. Finally, personalized dosimetry is likely to improve the efficacy and safety of radio- embolization in clinical practice and prospective studies with utilization of voxel-based dosimetry are necessary.

\section{Conclusion}

Although there have been many advances in the treatment of breast cancer, the prognosis for patients with metastatic 
disease remains poor and treatment is primarily palliative. Radioembolization has emerged as a viable therapy for patients with liver-dominant MBC who have preserved liver function and a good performance status. While there are many unanswered questions, it has been shown to be safe, effectively kill tumor, potentially increase survival, preserve quality of life, and allow for hepatic control without changing systemic therapy. Further investigation is clearly needed to help personalize treatment of these patients and optimize liver-directed therapy in conjunction with systemic therapy.

\section{Funding}

A.D. was funded by BTG, Dova Pharmaceuticals, and Sirtex Medical.

Conflict of Interest

None declared.

\section{References}

1 American Cancer Society (ACS) Cancer Facts \& Figures 2019. Atlanta: American Cancer Society; 2019

2 Howlader N, Noone AM, Krapcho M, et al, eds. SEER Cancer Statistics Review, 1975-2016, National Cancer Institute. Bethesda, MD. Accessed March 10, 2021 at: https://seer.cancer.gov/csr/1975_2016/

3 Harris JR. Cancer of the breast. In: DeVita VT, Hellman S, Rosenberg SA, eds. Cancer: Cancer Principles and Practice of
Oncology. Philadelphia, PA: J.B. Lippincott Company; 1993: 1264-1332

4 O'Shaughnessy J. Extending survival with chemotherapy in metastatic breast cancer. Oncologist 2005;10(Suppl 3):20-29

5 Adam R, Aloia T, Krissat J, et al. Is liver resection justified for patients with hepatic metastases from breast cancer? Ann Surg 2006;244(06):897-907, discussion 907-908

6 Leung AM, Vu HN, Nguyen KA, Thacker LR, Bear HD. Effects of surgical excision on survival of patients with stage IV breast cancer. J Surg Res 2010;161(01):83-88

7 Xie J, Xu Z. A population-based study on liver metastases in women with newly diagnosed breast cancer. Cancer Epidemiol Biomarkers Prev 2019;28(02):283-292

8 Liedtke C, Kolberg HC. Systemic therapy of advanced/metastatic breast cancer-current evidence and future concepts. Breast Care (Basel) 2016;11(04):275-281

9 Chua TC, Saxena A, Liauw W, Chu F, Morris DL. Hepatic resection for metastatic breast cancer: a systematic review. Eur J Cancer 2011;47(15):2282-2290

10 Cholapranee A, van Houten D, Deitrick G, et al. Risk of liver abscess formation in patients with prior biliary intervention following yttrium-90 radioembolization. Cardiovasc Intervent Radiol 2015; 38(02):397-400

11 Gabr A, Riaz A, Johnson GE, et al. Correlation of Y90-absorbed radiation dose to pathological necrosis in hepatocellular carcinoma: confirmatory multicenter analysis in 45 explants. Eur J Nucl Med Mol Imaging 2021;48(02):580-583

12 Davisson NA, Bercu ZL, Friend SC, et al. Predictors of survival after yttrium-90 radioembolization of chemotherapy-refractory hepatic metastases from breast cancer. J Vasc Interv Radiol 2020;31 (06):925-933 